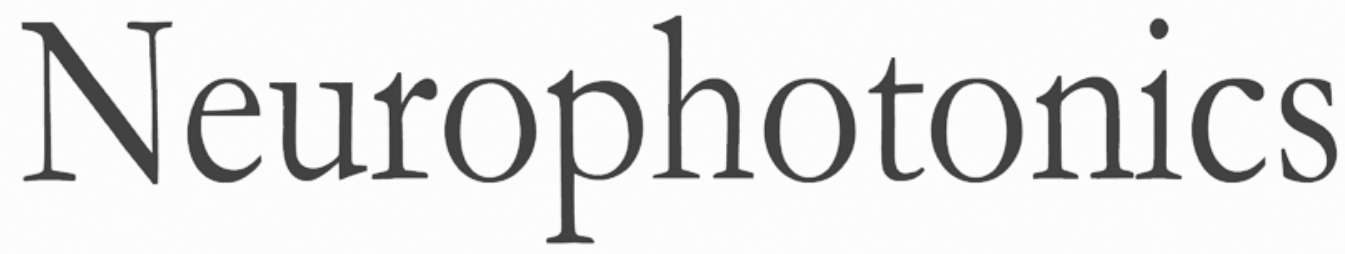

\title{
Functional near-infrared spectroscopy-informed neurofeedback: regional-specific modulation of lateral orbitofrontal activation and cognitive flexibility
}

Keshuang Li

Yihan Jiang

Yilong Gong

Weihua Zhao

Zhiying Zhao

Xiaolong Liu

Keith M. Kendrick

Chaozhe Zhu

Benjamin Becker 


\title{
Functional near-infrared spectroscopy-informed neurofeedback: regional-specific modulation of lateral orbitofrontal activation and cognitive flexibility
}

\author{
Keshuang Li, ${ }^{a}$ Yihan Jiang, ${ }^{\text {b }}$ Yilong Gong, ${ }^{\text {b }}$ Weihua Zhao, ${ }^{\text {a }}$ Zhiying Zhao, ${ }^{\text {a }}$ Xiaolong Liu, ${ }^{\text {a }}$ Keith M. Kendrick, ${ }^{a}$ \\ Chaozhe Zhu, b,c,d,* and Benjamin Becker ${ }^{a, *}$ \\ aUniversity of Electronic Science and Technology of China, The Clinical Hospital of Chengdu Brain Science Institute, MOE Key Laboratory \\ for Neuroinformation, Chengdu, China \\ ${ }^{\mathrm{b}}$ Beijing Normal University, State Key Laboratory of Cognitive Neuroscience and Learning, Beijing, China \\ ${ }^{\circ} B e i j i n g$ Normal University, IDG/McGovern Institute for Brain Research, Beijing, China \\ ${ }^{\mathrm{d} B e i j i n g ~ N o r m a l ~ U n i v e r s i t y, ~ C e n t e r ~ f o r ~ C o l l a b o r a t i o n ~ a n d ~ I n n o v a t i o n ~ i n ~ B r a i n ~ a n d ~ L e a r n i n g ~ S c i e n c e s, ~ B e i j i n g, ~ C h i n a ~}$
}

\begin{abstract}
Cognitive flexibility and reward processing critically rely on the orbitofrontal cortex (OFC). Dysregulations in these domains and orbitofrontal activation have been reported in major psychiatric disorders. Hemodynamic brain imaging-informed neurofeedback allows regional-specific control over brain activation and thus may represent an innovative intervention to regulate orbitofrontal dysfunctions. Against this background the present proof-of-concept study evaluates the feasibility and behavioral relevance of functional near-infrared spectroscopy (fNIRS)-assisted neurofeedback training of the lateral orbitofrontal cortex (IOFC). In a randomized sham-controlled between-subject design, 60 healthy participants have undergone four subsequent runs of training to enhance the IOFC activation. Training-induced changes in the IOFC, attentional set-shifting performance, and reward experience have served as primary outcomes. Feedback from the target channel significantly increases the regional-specific IOFC activation over the four training runs in comparison with sham neurofeedback. The real-time OFC neurofeedback group demonstrates a trend for faster responses during the set-shifting relative to the sham neurofeedback group. Within the real-time OFC neurofeedback group, stronger traininginduced IOFC increases are associated with higher reward experience. The present results demonstrate that fNIRS-informed neurofeedback allows regional-specific regulation of IOFC activation and may have the potential to modulate the associated behavioral domains. As such fNIRS-informed neurofeedback may represent a promising strategy to regulate OFC dysfunctions in psychiatric disorders. $\odot$ The Authors. Published by SPIE under a Creative Commons Attribution 4.0 Unported License. Distribution or reproduction of this work in whole or in part requires full attribution of the original publication, including its DOI. [DOI: 10.1117/1.NPh.6.2.025011]
\end{abstract}

Keywords: orbitofrontal cortex; neurofeedback; functional near-infrared spectroscopy; cognitive flexibility; neuromodulation; reward. Paper 19001RRR received Jan. 7, 2019; accepted for publication May 13, 2019; published online Jun. 8, 2019.

\section{Introduction}

Neurofeedback (NF) techniques have gained increasing attention as noninvasive means to regulate brain function for scientific and therapeutic purposes. ${ }^{1,2}$ The corresponding methods employ a biofeedback approach that uses real-time information of brain activity to enable self-regulation of a particular neural signal. ${ }^{1,3}$ Compared with the traditionally used electroencephalography NF, the use of hemodynamic imaging signals as near-real-time neural feedback is relatively new. The majority of hemodynamic NF studies employ functional magnetic resonance imaging (fMRI) techniques ${ }^{1,3}$ and a growing number of studies have demonstrated that fMRI NF-guided regulation of regional brain activity can produce changes in cognitive and emotional processes specifically associated with the target brain region. ${ }^{1,2,4}$ Based on accumulating evidence for the behavioral relevance of the NF-induced modulation of brain function,

*Address all correspondence to Benjamin Becker, E-mail: ben_becker@ gmx.de; Chaozhe Zhu, E-mail: czzhu@ bnu.edu.cn initial studies evaluated the potential of fMRI NF as a promising innovative intervention for psychiatric disorders. ${ }^{5}$

Although the therapeutic potential of fMRI-guided NF has been documented in initial randomized controlled trials, ${ }^{6,7}$ translation into clinical applications is hampered by the high costs of MRI assessments and the rather stressful MRI environment, as well as the limitations inherent to the MRI method, particularly a high sensitivity for motion and physiological noise, ${ }^{8}$ and field inhomogeneities caused by different magnetic susceptibilities to air and tissue resulting in signal loss in orbitofrontal regions. ${ }^{9}$ A previous fMRI NF study demonstrated that optimized MRI imaging parameters can improve signal quality in the orbitofrontal cortex (OFC) ${ }^{10}$ however, imaging orbitofrontal regions-particularly under real-time signal-processing conditions-remains challenging.

Functional near-infrared spectroscopy (fNIRS) is a noninvasive optical neuroimaging technique that-similar to blood oxygen-level-dependent MRI—can be employed to detect changes in hemoglobin concentration associated with neural activity. ${ }^{11}$ Briefly, neural metabolism is supported through a localized 
vascular response that causes an influx of oxygen-rich blood to the active region, reflected by a regional increase in oxyhemoglobin (oxy-Hb) and a decrease in deoxyhemoglobin (deoxy-Hb). ${ }^{12}$ fNIRS measures the concentration of oxy-Hb and deoxy-Hb in cerebral vessels according to their absorption spectra for light in the near-infrared range. ${ }^{13}$ Despite limitations, particularly a restricted penetration depth and resolution, fNIRS has been increasingly employed in cognitive and clinical neuroscience. Owing to the recent technological and methodological progress in fNIRS imaging and advantages over fMRI, including lower costs, easy application, and robustness against motion and susceptibility artifacts, fNIRS has become an attractive hemodynamic imaging alternative.

The OFC, a ventral subdivision of the prefrontal cortex, is a cytoarchitectonically and functionally heterogeneous region with dense connections to cortical and subcortical areas, including sensory regions as well as limbic and striatal regions. ${ }^{14}$ Supported by the subregion-specific segregated circuits, the OFC contributes to several highly integrative functional domains, including emotion, decision-making, value coding, and behavioral flexibility. ${ }^{15,16}$ Across species, lesions of the OFC produce marked impairments in reversal learning and attentional set-shifting, suggesting a critical role of the OFC in cognitive flexibility. ${ }^{15}$ Studies employing reversal learning paradigms reported that rodents with OFC damage could learn the initial discrimination by responding to one cue to receive reward and to withhold or inhibit a response to avoid punishment or nonreward. However, after cue-outcome associations are reversed, OFC-lesioned rodents required considerably longer to adapt their behavior. ${ }^{17-22}$ More recently, the role of the OFC in reversal learning has additionally been confirmed by human fMRI studies. These studies used rewarding and punishing stimuli and reported that the adaptation to changing reinforcement contingencies is mediated by the OFC. Cognitive flexibility additionally encompasses attentional set-shifting, supporting flexible behavioral adaptation in the context of relevant and irrelevant information. Set-shifting paradigms have been combined with fMRI to dissect regional-specific contributions of the prefrontal cortex to cognitive flexibility subdomains, and it has been reported that the attentional control sub-facet engages the ventrolateral cortex, whereas reversals specifically engage the lateral $\mathrm{OFC}{ }^{23}$ In addition to cognitive flexibility, the role of the OFC in reward processing, specifically modulating outcome expectancies related to reward, has been well documented. For instance, human neuroimaging studies have implicated the OFC in the anticipation and evaluation of expected outcomes ${ }^{24}$ as well as value-guided decision-making. ${ }^{25}$

In line with the important role of the OFC in cognitive flexibility and reward processing, neural alterations in this region have been consistently reported in psychiatric disorders characterized by dysregulations in these domains, most prominently addictive disorders, obsessive compulsive disorder, ${ }^{15}$ attentiondeficit/hyperactivity disorder, ${ }^{26}$ and major depression. ${ }^{27}$ Given that the efficacy of the established pharmacological and behavioral treatment options for these disorders is limited, ${ }^{28}$ it has been advocated to employ hemodynamic imaging-informed $\mathrm{NF}$ to modulate the identified neural alterations in a regionalspecific manner to alleviate psychiatric symptoms and normalize functional deficits. ${ }^{28-31}$

To facilitate translation of hemodynamic NF into clinical applications, the present randomized, sham-controlled study aims at evaluating the feasibility to employ fNIRS-informed
NF as strategy to modulate brain activity. Initial studies have demonstrated the feasibility to use fNIRS-informed NF to allow participants to acquire regulatory control over brain activity in motor-related regions. ${ }^{32,33}$ Subsequent studies have demonstrated the feasibility to gain regulatory control over prefrontal brain activity via fNIRS-informed NF and its potential to enhance executive functions. ${ }^{34}$ Given the important contribution of OFC dysregulations to the functional impairment in psychiatric patients as well as the methodological and practical challenges to use fMRI-based NF approaches in this context, the present fNIRS NF study targets the lateral OFC. Importantly, previous studies have shown that fNIRS can sensitively detect both deoxy-Hb and oxy-Hb changes in the OFC and can reliably assess neural activity in the OFC during cognitive flexibility and reward evaluation. ${ }^{35-37}$ Moreover, an increasing number of studies have employed fNIRS imaging to associate aberrant cognitive flexibility and reward processing with altered OFC activation in psychiatric patients. ${ }^{38}$ To further determine the functional relevance of the NF-induced OFC activation changes, behavioral indices of cognitive flexibility and reward processing-both of which have been associated with the lateral $\mathrm{OFC}^{15}$ - serve as primary behavioral outcomes to evaluate the training success. To control the unspecific effects of training, the fNIRS-informed OFC NF is embedded in a randomized shamcontrolled between-subject experiment with a total of $n=60$ healthy participants. Based on previous studies, ${ }^{4,6}$ we expected that participants in the real-time OFC NF group, but not in the sham NF group, would learn to successfully upregulate regional-specific activity in the lateral OFC and that this would be accompanied by increased cognitive flexibility and reward experience on the behavioral level.

\section{Methods and Materials}

\subsection{Participants}

A total of $n=60$ healthy young males were enrolled in the present study. The main aim of the study was to determine the feasibility and functional relevance of real-time fNIRS-informed $\mathrm{NF}$ training targeting the lateral OFC. To reduce variance related to sex differences or effects of menstrual cycle on OFC activity and the primary behavioral outcomes, ${ }^{39}$ the present proof-ofconcept experiment focused on male participants. To control for the unspecific effects of the training procedures on the primary outcomes, the NF training was embedded in a randomized, sham-controlled between-subject experimental design. Participants were randomly assigned (30 participants in each group) to receive either real-time feedback from the OFC target region (real-time OFC NF group) or a sham feedback (sham NF group) during the training. Participants were randomized without stratifying for further variables. All participants provided written informed consent. The study had full ethical approval by the local ethics committee of the University of Electronic Science and Technology of China, and the procedures were in accordance with the latest version of the Declaration of Helsinki.

\subsection{Neurofeedback Training Protocols and Procedures}

The training session included four subsequent runs of alternating rests and regulation blocks (four blocks per run, block duration being $25 \mathrm{~s}$ ). The real-time OFC NF group received 


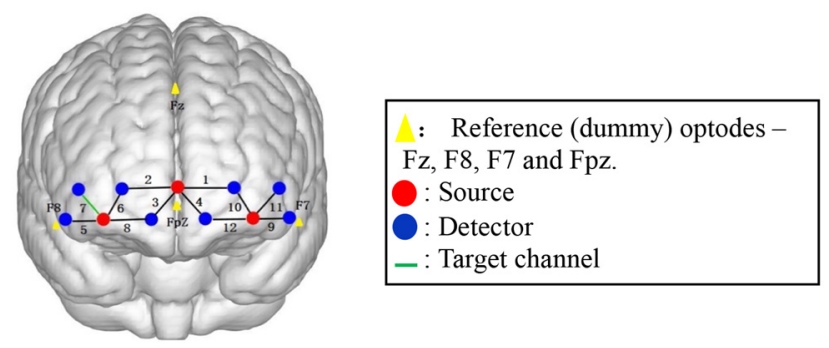

Fig. 1 Optodes configuration (three sources: red dots, eight detectors: blue dots) and placement. The optodes are placed in line with the International 10-20 system using the Fz, F7, F8, and Fpz as anchor points (visualized as yellow triangles). Source and detectors are positioned $30-\mathrm{mm}$ apart. A black line connecting a detector and a source represents a measurement channel and the corresponding channel number. Six channels are used per hemisphere: two channels covering the medial OFC (right hemisphere: channels 2 and 3; left hemisphere: channels 1 and 4 ) and four channel covering the lateral OFC (right hemisphere: channels 5 to 8 ; left hemisphere: channels 9 to 12). The source in the middle of the probe array is located on the Fpz. Optode placement is anatomically symmetrical on both hemispheres. Channel 7 (visualized as green line) serves as a predefined online feedback channel during the NF training.

real-time feedback from fNIRS channel 7 located over the right lateral OFC (locations of the optodes and feedback channel are displayed in Fig. 1), whereas the sham NF group received feedback from a participant who had previously underwent the experimental training (similar approach in Kober et al. ${ }^{32}$ ). To accustom the participants to the equipment and to reduce variance related to trial-and-error attempts during the initial training blocks, the training was preceded by a pretraining session during which all participants received real-time OFC NF and were required to explore a suitable regulation strategy during 6 to 10 feedback blocks. When participants reported that they had discovered an effective strategy, a recovery break of 10 to $30 \mathrm{~min}$ was included before the training session started (protocols are displayed in Fig. 2). Participants were asked to employ the strategies they discovered during the pretraining session to increase the lateral OFC activity during the training.

\subsection{Feedback and Training Condition Visualization}

The online feedback was visually displayed to the participants by a stone in front of a beach landscape (see Fig. 2). The height the stone floats over the ground visualized the neural activity level in the chosen feedback channel 7 (the higher the stone floats over the ground, the higher the activity). Participants were asked to lift the stone as high as possible by regulating their brain activity by using a mental strategy. We decided to embed the feedback in this "stone-lifting" game rather than using a simple thermometer visualization to increase the motivation of the participants during the training. The conditions (rest/regulate) were visually presented to the participant via two lights on top of the display screen (see Fig. 2). The rest (instruction, relax) condition was indicated by a red light on the left, the regulate (instruction, lift the stone) condition was indicated by a green light on the right.

\subsection{Instructions for the Participants}

The participants were asked to try to lift the stone as high as possible by regulating their brain activity. Participants were informed that the purpose of the training was to examine whether they could learn to upregulate their OFC activity. To increase their regulation ability the neural regulation success would be visually presented to them via the stone-lifting game (all participants were explicitly informed that the higher the stone floats the higher is the OFC regulation success). Given that an explicit strategy instruction is not necessary for successful NF-assisted acquisition of neural regulation, ${ }^{40,41}$ no explicit strategies for regulation were provided to the participants. Participants were instructed not to control the height of the stone by physical means, such as breathing or head/body motion but rather to discover efficient mental control strategies. Once they discovered an efficient strategy to lift the stone during the pretraining, they were asked to continue using the strategy during the subsequent training sessions.

\subsection{Post-Training Behavioral Assessments: Cognitive Flexibility and Reward Experience}

To determine the functional relevance of the training on the behavioral level, the participants were administered the intra-extra

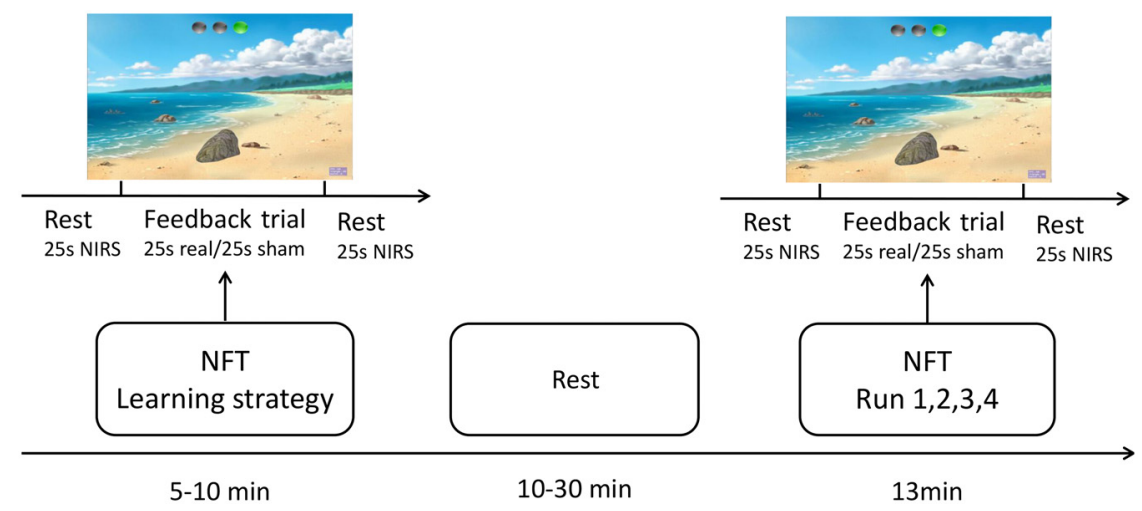

Fig. 2 Experimental procedures for the two training sessions. The online feedback is visually embedded in a "stone-lifting" game. The height of the stone floating over the ground visualizes the activity in the target channel (higher oxy- $\mathrm{Hb}$ activity in channel $7=$ increase in the height of the stone). The experimental conditions (rest/regulate) are visually presented via two lights on top of the display screen $($ red $=$ rest; green $=$ regulate $)$. 
dimensional set shift (IED) task and rated the rewarding experience of the training on a visual analogue scale after the training session. The IED paradigm has been widely employed to examine cognitive flexibility and has a high sensitivity for changes in frontostriatal functioning. ${ }^{42}$ In this task, participants are required to use the provided feedback to discover a rule that determines which stimulus is correct. After six correct responses, the stimuli and/or rule changes. Initially the task involves simple stimuli (two different pink shapes); during this stage, shifts in the rule are intradimensional. During later stages of the task, compound stimuli are used (e.g., white lines overlay the pink shapes); corresponding shifts refer to extra-dimensional shifting. To assess effects of training on reward experience participants were additionally asked to rate their liking of the training on a 1 to 9 scale ("How much did you enjoy the task").

\subsection{NIRS Data Acquisition, Online Preprocessing, and Neurofeedback}

Hemodynamic response (HR) signals were assessed using a NIRSport fNIRS Systems (eight sources/eight detectors, NIRx Medizintechnik, GmbH, Berlin, Germany) operating at two wavelengths (760 and $850 \mathrm{~nm}$ ) at a sampling rate of $20.83 \mathrm{~Hz}$. An optode set of 3 sources and 8 detectors was used leading to 12 source-detector pairs (channels; see Fig. 1, optode placement in line with Ieong and Yuan ${ }^{43}$ ). Optodes were accurately placed according to the International $10-20$ system, $^{44}$ and the sourcedetector distance was $30 \mathrm{~mm}$. In line with a previous fNIRS study, the channels were allocated to the medial and lateral regions of the OFC (in line with Leong and Yuan, ${ }^{43}$ channels $1-4$ of the current study would be allocated to the medial OFC, whereas the other channels would be allocated to the lateral OFC). Channel 7 was designated as right lateral OFC feedback channel and the position of the channel was further validated by MRI scans in two independent participants (see Fig. S1 in Supplementary Material).

In line with previous fNIRS NF studies, the feedback was based on the oxy-Hb signal. ${ }^{45-47}$ Online preprocessing of the oxy-Hb NIRS signal was performed by the built-in real-time output solution implemented in the NIRSport system. Next, the real-time output was computed and visually displayed via a previously evaluated real-time fNIRS NF platform. ${ }^{48}$ The raw oxy-Hb NIRS signal was smoothed using a 2-s moving average window. A baseline was calculated by taking the average of signals $2 \mathrm{~s}$ before each regulation block and was subsequently subtracted from the smoothed signal. The feedback signal was computed in real-time from the signal of the a priori target channel (No. 7) using the equation "feedback signal = preprocessed oxy-hemoglobin signal/difficult coefficient."

The "difficulty coefficient" served to calibrate the feedback visualization according to the signal range in the current study to promote learning success (e.g., feedback visualization that only spans a small range of the thermometer used for feedback display may not provide sufficient information for the participants to learn the regulation). The study-specific "difficulty coefficient" was therefore initially determined in a pre-experiment that was conducted before the start of the primary experiment that examined the feasibility of OFC NF. During this pre-experiment, an independent sample of $n=6$ participants underwent four runs of the OFC NF training. Each run used a separate difficulty coefficient $(0.003,0.006,0.008,0.01$ values based on our experience during implementing the paradigm). The order of the four runs with the separate difficulty coefficient was randomized across participants. Subsequently, participants were asked to designate the run with the most suitable difficulty to allow regulation. Four (from six participants) designated the run that used 0.008 ; consequently, this coefficient was employed to visualize the online feedback in terms of the height of the stone for all participants in the subsequent study. Participants in the sham $\mathrm{NF}$ group received oxy-Hb signal changes from a participant in the real-time OFC NF group who had previously completed the training. Participants in both groups were blinded for the training condition they received and the experimenter operating the feedback platform was separated from the participant by a partition wall to minimize any interaction during pretraining and training.

\subsection{Offline Preprocessing and Analyses}

The fNIRS raw data were preprocessed and analyzed using the NIRS toolbox in SPM (Statistical Parametric Mapping $\left.{ }^{49}\right)^{50}$ and in-house scripts in MATLAB (The MathWorks, Inc.). During preprocessing, a second-order detrending was applied to remove the baseline drifts and low-pass filtering [Gaussian smoothing with full width at half maximum (4 s)] was employed to remove high-frequency noise. To control potential confounding effects of head motion during training, motion correction, as implemented in the NIRS analysis package (NAP), was applied to the offline data. ${ }^{51}$ The subsequent data analysis focused on the oxy$\mathrm{Hb}$ signal as it has been demonstrated to exhibit larger signal changes and higher sensitivity to task-related neural activation compared to the deoxy-Hb signal. ${ }^{52-54}$ A generalized linear model (GLM) approach was employed to model the task-related HR on the individual level including the regulation periods modeled by a boxcar function while the rest periods were included as implicit baseline. Serial autocorrelations in the GLM analysis were accounted for by employing prewhitening as incorporated in NIRS SPM [AR(1) model, in line with recommendations by Huppert ${ }^{55}$. The first-level design matrix was convolved with the standard HR function, as provided in NIRS SPM.

For the group-level analyses, beta estimates were obtained for each participant and channel. The primary outcome to determine the training success on the neural level was oxy-Hb change over the training runs in the target channel (right lateral OFC; channel 7). To further control the unspecific effects of training or effects of mental effort on OFC activity, individual-level beta values from the target channel were subjected to group-level activation analyses comparing the real-time OFC NF and the sham NF group. Differences were considered significant using a channel-level threshold of $p<0.05$ (Bonferroni-corrected).

\subsection{Primary Outcomes and Evaluation of Training Success}

Training-induced oxy-Hb changes in the OFC target channel served as primary outcome to evaluate the training success on the neural level. Channel-specific activity beta estimates were employed as dependent variables, and effects of training were determined by means of mixed analysis of variance (ANOVA) models including the between-subject factor group (real feedback versus sham feedback) and the within-subject factor training run (run1/run2/run3/run4). Significant effects were further explored by means of appropriate Bonferronicorrected post-hoc tests. To evaluate the functional relevance of training on the behavioral-level, post-training IED performance indices and liking ratings were compared between the two 
training groups. Associations between neural and behavioral training success were explored by means of analyzing correlations between training-induced $1 \mathrm{OFC}$ oxy-Hb changes (run4 > run1) and behavioral indices within the training groups. Statistical analyses were carried out using SPSS version 22.0 (IBM, Inc.).

\subsection{Additional Exploratory Analyses}

To further explore whether the training led to regional-specific activity changes in the region that served as real-time NF source (right lOFC) or rather unspecific increases in OFC activity, an exploratory ANOVA was performed. This ANOVA employed the dependent variable channel-specific beta estimates and the between-subject factor group (real feedback versus sham feedback) and the within-subject factors training run (run1/run2/ run3/run4) and channels (channels 1 to 12). Moreover, an additional exploratory analysis examined training-related changes in the deoxy-Hb signal of the primary outcome channel 7. To this end, a mixed ANOVA with the within-subject factor run (run1/ run2/run3/run4), the between-subject factor group (real feedback versus sham feedback), and the dependent variable beta values from channel 7 was performed.

\subsection{Upregulation Strategies}

After the training, all participants were asked to report the strategies they employed during the training. The reported strategies were qualitatively assessed by six independent male raters. To control for different strategies between the training groups, the frequencies of the reported regulation strategies were compared between the real-time OFC NF group and the sham NF group using Pearson $\chi^{2}$ test. $^{56}$

\subsection{Control of Potential Confounders}

To further control confounding effects of pretraining betweengroup differences in mood and psychopathological symptom load, corresponding indices were assessed by means of the positive and negative affect schedule, ${ }^{57}$ state anxiety inventory, ${ }^{58}$ Beck depression inventory-II, ${ }^{59}$ and Barratt impulsiveness scale ${ }^{60}$ administered before the training sessions. To further control potential confounding effects of between-group differences in the perceived training success, all participants were required to rate their training success (scale ranging from -4 to 4 ).

\section{Results}

\subsection{Data Quality Control}

Two participants reported that they were unable to gain control over their brain activity in the pretraining test runs and were thus excluded from the subsequent NF training. Initial examination of the primary neural outcome (oxy-Hb) data quality identified one participant from each group as an outlier with respect to data from the training channel during at least two runs (outliers were defined as subjects that deviated larger than two standard deviations from the run-specific group mean, outliers were additionally confirmed using the SPSS outlier detection function). Consequently, oxy- $\mathrm{Hb}$ data from these participants were excluded from all analyses resulting in a total of $n=56$ participants for the primary analysis $(n=27$, real-time OFC NF group; $n=29$ sham NF group).

\subsection{Assessment of Confounders: Age, Mood, and Psychopathology}

The training groups did not differ with respect to age $\left(M_{\text {real }}=\right.$ 21.3 years, $\mathrm{SD}=2.02 ; \quad M_{\text {sham }}=21.7$ years, $\mathrm{SD}=2.08$; $t=-0.656, p=0.515$ ) or pretraining mood and psychopathological load (Table 1, $t<1.463$, ps $>0.149$ ). Moreover, the training groups reported a comparable evaluation of their perceived training success $(t=1.009, p=0.317)$.

\subsection{Evaluation of Training Success-Primary Neural Outcome}

A mixed two-way ANOVA with the factors run (run1/run2/run3/ run4) and group (real feedback versus sham feedback) and the dependent variable lateral OFC activity as measured by the beta values (oxy- $\mathrm{Hb})$ from the target channel revealed a main effect of run $\left[F_{(3,162)}=7.26, p=0.001\right]$ and group $\left[F_{(1,54)}=15.52\right.$, $p<0.001]$ as well as a run $\times$ group interaction effect $\left[F_{(3,162)}=5.25, p=0.005\right]$. Post-hoc comparisons demonstrated that activation in the target channel significantly increased over the course of the real feedback training (run $1<$ run3, $p<0.001 ; \quad \operatorname{run} 1<\operatorname{run} 4, \quad p=0.003 ; \quad \operatorname{run} 2<\operatorname{run} 3$, $p=0.001 ; \operatorname{run} 2<\operatorname{run} 4, p=0.013$, two-tailed). Concordant analysis of the sham training data did not yield significant changes in the target channel (all $p s>0.141$, two-tailed). Directly comparing the training groups further revealed that the real-time OFC NF group exhibited significantly higher activity during runs 3 and 4 ( $p<0.001$, two-tailed) in the lateral OFC target channel as compared to the sham NF group; however, the group did not exhibit higher activity during runs 1 and 2 ( $p>0.071$, two-tailed, see Fig. 3), thus further confirming training success on the neural level.

For further data quality assessment and to increase the transparency representative time courses for one participant per group (real feedback versus sham feedback), both online data after corrected for motion-related artifacts and offline data after preprocessing are visualized in Fig. 4. Figure 4 displays the time courses for run4; data for all runs is furthermore provided in Figs. S2-S5 in Supplementary Material.

Table 1 Pretraining mood and psychopathological symptom load in the two training groups, mean and SDs (in brackets) are reported.

\begin{tabular}{lccc} 
& $\begin{array}{c}\text { Real-time OFC } \\
\text { NF group } \\
(N=27)\end{array}$ & $\begin{array}{c}\text { Sham } \\
(N=29)\end{array}$ & $\begin{array}{c}\text { Two sample } \\
\text { t-test } \\
(p \text {, two-tailed })\end{array}$ \\
\hline PANAS-P & $26.19(6.86)$ & $26.21(5.68)$ & 0.990 \\
PANAS-N & $16.78(7.01)$ & $14.41(4.98)$ & 0.149 \\
SAI & $38.67(8.49)$ & $36.83(7.45)$ & 0.392 \\
BDI II & $8.00(7.23)$ & $6.79(6.46)$ & 0.512 \\
BIS (Attention) & $14.11(2.49)$ & $13.79(3.05)$ & 0.672 \\
BIS (Motor) & $18.78(3.34)$ & $18.45(3.46)$ & 0.719 \\
BIS (Planning) & $23.59(3.80)$ & $24.3793(4.19)$ & 0.466 \\
\hline
\end{tabular}

Note: PANAS-P, positive and negative affect schedule-positive mood; PANAS-N, positive and negative affect schedule-negative mood; SAI, state anxiety inventory; BDI II, Beck depression inventory-II, BIS, Barratt impulsiveness scale. 
Real-time OFC NF group

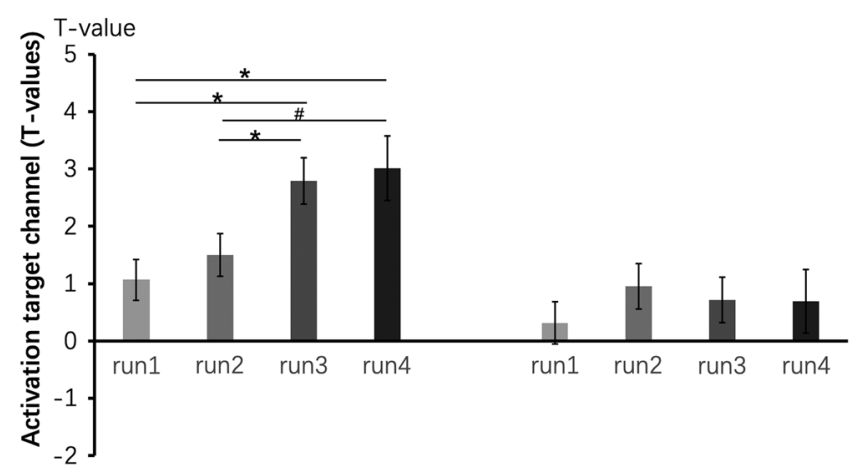

Fig. 3 The oxy-Hb signal in the target channel significantly increases over the course of the real-time OFC NF training runs but not during the sham NF training. Differences between the training runs are tested by posthoc paired $t$-tests, two tailed; ${ }^{*} p<0.0125$; \# denotes marginal significance, $p<0.05$.

\subsection{Exploratory Analysis-Regional Specificity of the Training Effects}

To explore the regional specificity of the training, the effect of training on all OFC channels was explored. A mixed ANOVA with the factors run (run1/run2/run3/run4), group (real feedback versus sham feedback), and channels (channels 1 to 12) and the dependent variable OFC activity as measured by the oxy-Hb beta values revealed a main effect of run $\left[F_{(3,162)}=3.96, p=\right.$ $0.015]$, a main effect of channel $\left[F_{(11,594)}=6.52, p<0.001\right]$, and a channel $\times$ group interaction effect $\left[F_{(11,594)}=4.35\right.$, $p=0.001]$, but no main effect of group and no other interaction effects. To control for multiple comparisons, a Bonferroni correction was used to account for all channels tested. Post-hoc comparisons demonstrated that the significant differences between the real-time OFC NF group and the sham NF group were observed in the target channel 7 ( $p<0.001$, two-tailed) and the adjacent channel 6 ( $p=0.012$, two-tailed) in the right lateral OFC (for details, see Figs. 1 and 5). For all other channels the interaction effect was not significant (all $p s>0.12$, twotailed), indicating that training specifically modulated activity in the right lateral OFC.

\subsection{Exploratory Analysis-Training-Related Changes in Deoxy-Hemoglobin}

For offline processing of the deoxy-Hb signal, concordant procedures as for the oxy-Hb signal (including motion correction) were applied. Please note that the GLM analysis for the deoxy$\mathrm{Hb}$ data employed a negative HR function, whereas the oxy-Hb data was processed using a positive HR function (as implemented in NIRS SPM). Therefore, a positive $T$-value for the oxy-Hb signal reflects an increase in oxy-Hb, whereas a positive $T$-value for the deoxy-Hb signal reflects a decrease in deoxy-Hb. In line with the oxy-Hb analyses initial data quality assessments (a)

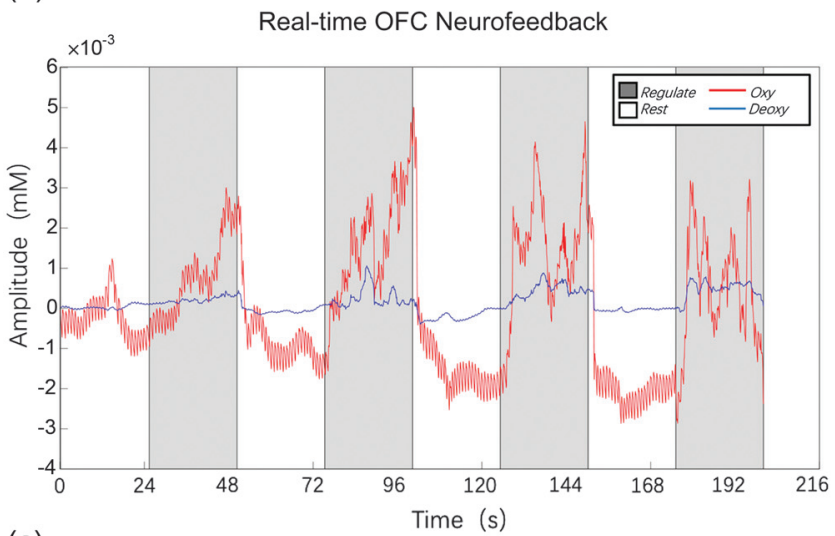

(c)

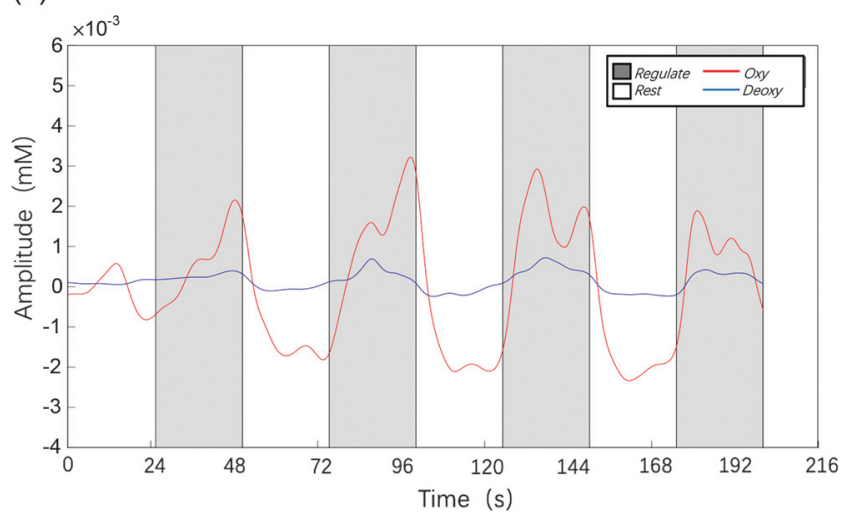

(b)

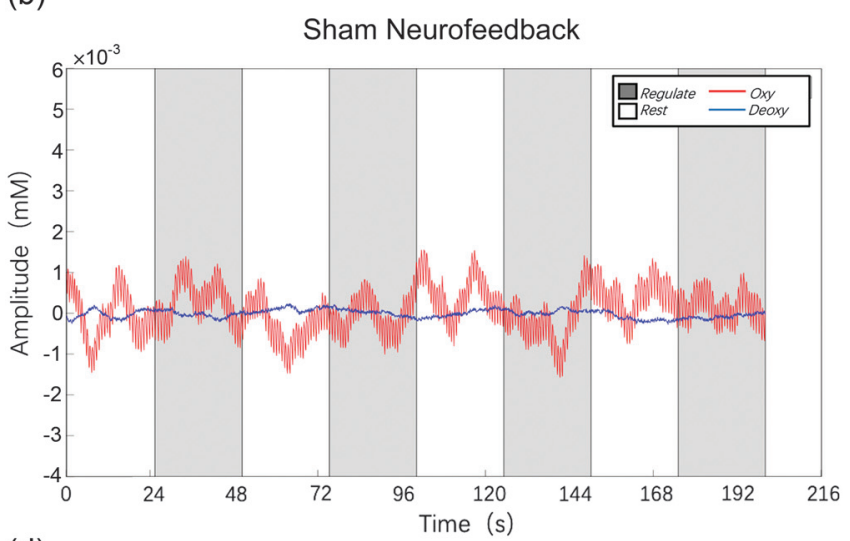

(d)

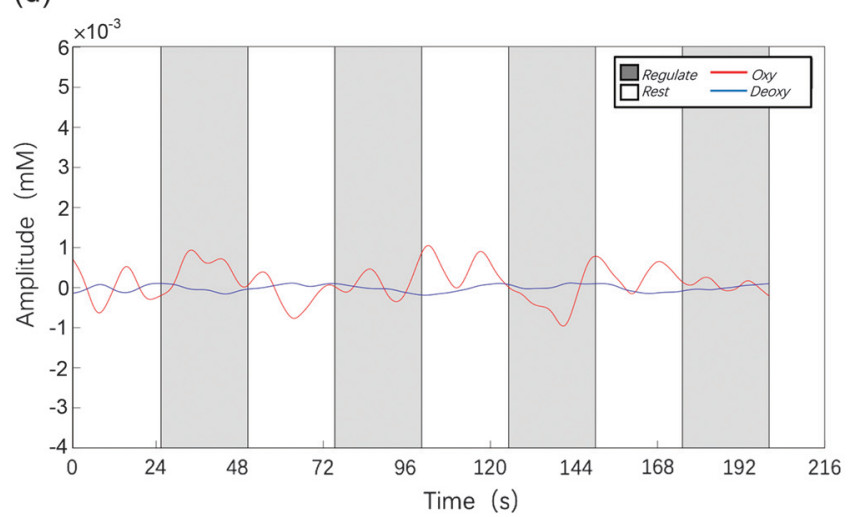

Fig. 4 Time courses of oxy- $\mathrm{Hb}$ (red) and deoxy- $\mathrm{Hb}$ (blue) for two representative participants (a), (c) from the same participant during real-time OFC NF; (b), (d) from the same participant during sham NF. Online preprocessed data are shown in the upper panel (corrected for motion-related artifacts); offline preprocessed data are shown in the lower panel. Rest periods are displayed on white background, and regulation periods are displayed on gray background. Only time courses for run 4 are displayed. 


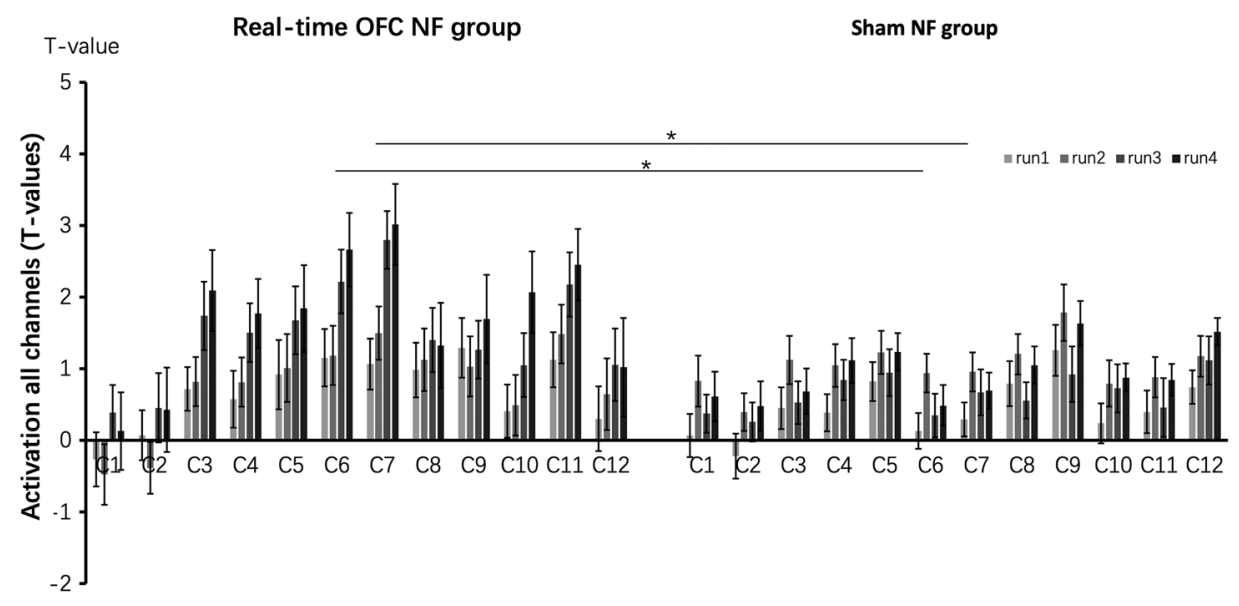

Fig. 5 Significant differences between the real-time OFC NF group and the sham NF group are observed in the target channel 7 (C7) and the adjacent channel 6 (C6) both in the right IOFC. Differences between groups are tested by means of posthoc two-sample $t$-tests, two tailed; ${ }^{*} p<0.004$ (Bonferroni corrected).

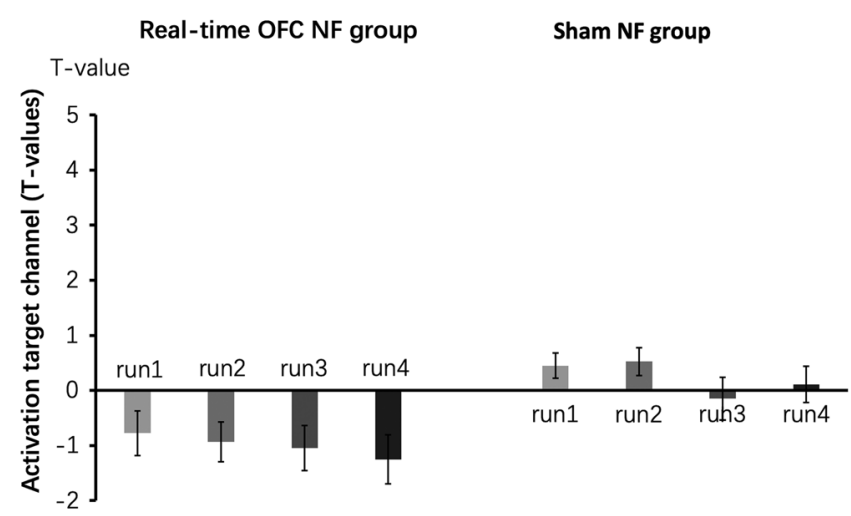

Fig. 6 Deoxy-Hb signal from the target training channel.

on the de oxy-Hb signal were performed. Applying identical criteria data from $n=3$ participants were excluded from the deoxy-Hb signal analyses $(n=1$ from the real-time OFC NF, $n=2$ from the sham NF group), leading to a final sample size of $n=55$ participants for the deoxy-Hb signal analysis $(n=27$, real-time OFC NF group; $n=28$, sham NF group). A mixed ANOVA with the factors run (run1/run2/run3/run4) and group (real feedback versus sham feedback) and the dependent variable beta values from the 1OFC target channel revealed a main effect of group $\left[F_{(1,53)}=11.09, p=0.002\right]$, but no main effect of run and no run $\times$ group interaction effect (both $p s>0.256$, see Fig. 6).

\subsection{Evaluation of Training Success-Primary Behavioral Outcome}

Performance indices for the IED task included the number of errors, the number of trials completed, the number of stages completed, and reaction times. In line with the previous research, the median reaction times were used as estimate of central tendency. ${ }^{61}$ Results revealed significant shorter reaction times for correct responses after the real-time OFC NF training compared to the sham NF training [ $p=0.037$, one-tailed, twosample $t$-test, see Fig. 7(a)]. Other indices of the IED task and rewarding experience failed to reach statistical significance (all $p>0.05$, one-tailed).

\subsection{Association between Behavioral and Neural Training Success}

A subsequent correlation analysis examined the association between behavioral (median reaction time in the IED task) and neural training success (changes run4 $>$ run1 activation in oxy$\mathrm{Hb}$ in target channel). Results revealed that reaction times for correct responses showed a descriptive, but not significant, negative association with OFC activity changes in the real-time OFC NF group $\left[r_{\text {real }}=-0.286, p=0.148\right.$, see Fig. 7(b)] but a marginal positive association in the sham $\mathrm{NF}$ group $\left[r_{\text {sham }}=0.359, p=0.056\right.$; stable after excluding one outlier, $r_{\text {sham }}=0.084, p=0.671$, significant correlation differences according to Fisher's $Z$ test, $z=-2.39, \quad p=0.017$; see Fig. 7(b)].

Given that a previous study reported significant associations between fNIRS-assessed OFC activity and liking levels, ${ }^{36}$ associations with post-training liking ratings were additionally explored. Results indicated that the liking level was significantly positively associated with $\mathrm{OFC}$ oxy-Hb changes in the real-time OFC NF group $\left[r_{\text {real }}=0.483, p=0.011\right.$, see Fig. 7(c)], but not in the sham NF group $\left[r_{\text {sham }}=0.024, p=0.901\right.$, marginal significant between-group correlation differences according to Fisher's $Z$ test, $z=1.80, p=0.073$; see Fig. 7(c)].

\subsection{Regulation Strategies Reported by the Participants}

In line with a previous study that evaluated regulation strategies during NF training with the present platform, ${ }^{56}$ the content analysis identified three main clusters of upregulation strategies: (1) imagination ("imagining I will reach a greater level of career success"), (2) experience recall ("thinking about my happy memories"); (3) meditation ("thinking of nothing particular and relaxing"). Importantly, the groups did not differ in the regulation strategies employed during the training (Pearson $\chi^{2}$ test, $p=0.734$, two-tailed, Table 2), arguing against confounding effects of different regulation strategies on the observed neural and behavioral between-group differences.

\section{Discussion}

The present proof-of-concept study evaluated the feasibility and functional relevance of real-time fNIRS-informed NF training as 
(a)

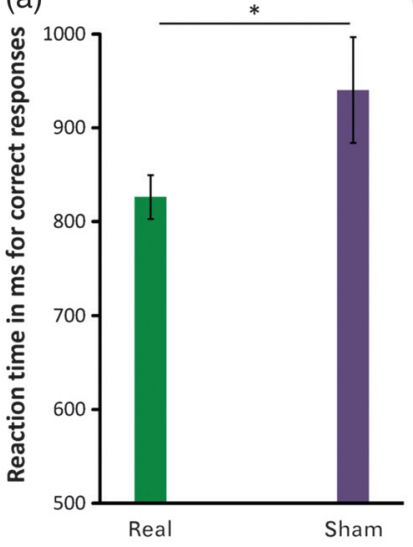

(b)

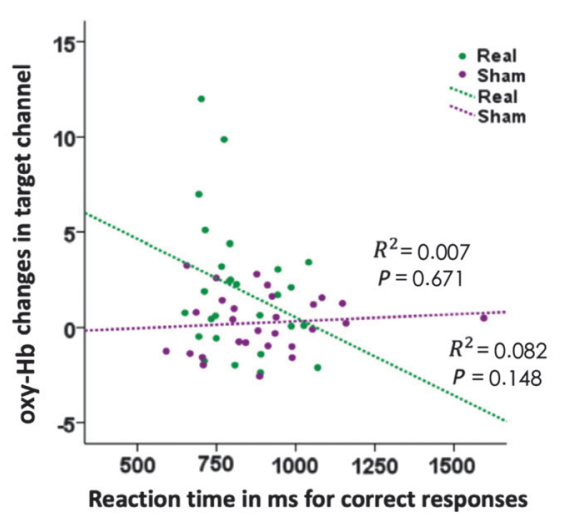

(c)

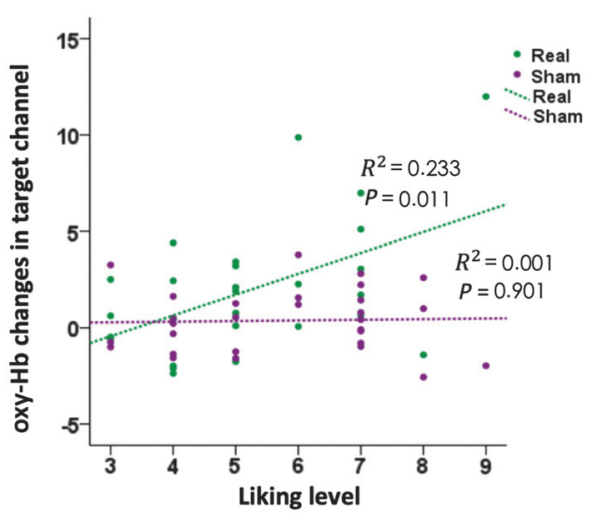

Fig. 7 (a) Significant differences between the real-time OFC NF group and the sham NF group are observed in the reaction time for correct responses in the IED paradigm. Between-group differences are tested by means of two-sample $t$-tests, one tailed; " $p<0.05$. (b) In the real-time OFC NF group, stronger training-induced IOFC activity changes (run4 > run1) are negatively associated with IED response times, whereas a positive association is observed in the sham NF group. (c) In the real-time OFC NF group, stronger IOFC changes are positively associated with higher levels of liking, and in the sham NF group, no association is observed.

Table 2 Regulation strategies reported by the participants.

Real-time OFC NF group

Sham NF group

\begin{tabular}{lcc}
\hline Imagination & 8 & 6 \\
Experience recall & 12 & 14 \\
Meditation & 7 & 9 \\
\hline
\end{tabular}

Note: Numbers correspond to the number of participants reporting the corresponding regulation strategy in each training group.

closed-loop strategy to increase 1OFC activation. Using a randomized sham-controlled between-subject design, the present study revealed that participants in the real-time OFC NF group successfully learned to increase $1 \mathrm{OFC}$ oxy-Hb activity over the course of four subsequent training runs. Importantly, no significant changes in neural activation were observed in the sham NF group. Together with the lack of between-group differences in perceived training success and regulation strategies, the lack of training-induced changes in the sham NF group argues against unspecific effects of the training procedure and emphasizes the specific importance of the feedback signal for successful acquisition of neural regulation. Exploring the regional specificity of the training effects on OFC activation revealed that significant training-induced oxy-Hb changes were restricted to the target and an adjacent channel, suggesting that the training produced regional-specific increases in right $1 \mathrm{OFC}$ activation. On the behavioral level, the real-time OFC NF group demonstrated a trend for enhanced cognitive flexibility as reflected by decreased response times for correct responses in the attentional set-shifting task. Moreover, exploratory analyses revealed that shorter response times and higher rewarding experience were associated with stronger training-induced oxy-Hb increases in lOFC activity, further confirming a potential functional relevance of successful $1 O F C$ regulation via fNIRS-informed NF.

Comparing the real-time OFC NF group with the sham NF group demonstrated that fNIRS-informed NF from the target channel allowed participants to acquire regulatory control over regional-specific activation in the 1OFC. Examining the activation changes within the groups further documented that IOFC activity significantly increased over the four training runs in the real-time OFC NF but not in the sham NF group. The successful upregulation in the real-time OFC NF group was further confirmed by between-group analyses showing significantly higher IOFC activity in the real-time OFC NF group compared to the sham NF group during the last two training runs. The present results add to the growing number of reports, suggesting that fNIRS-informed NF training allows participants to gain volitional control over cortical brain activity, including motor as well as frontal regions. ${ }^{32-34}$ Together with a previous study employing fMRI-informed NF to train participants to volitionally modulate orbitofrontal activity, ${ }^{10}$ the present findings additionally document that hemodynamic NF-assisted control over regional activation in the OFC is feasible. Compared to fMRIinformed NF, fNIRS-informed NF is limited by characteristics inherent to the acquisition methodology, particularly a lower spatial resolution and restricted signal acquisition from cortical regions. However, particularly for clinical applications or for targeting regions susceptible to MRI artifacts such as the OFC, the advantages of fNIRS-informed NF may outweigh the limitations and promote translation into the clinical practice. Orbitofrontal alterations have been demonstrated in several disorders characterized by deficits in cognitive flexibility and value processing, particularly attenuated OFC activation during impaired cognitive flexibility in pediatric and adult obsessivecompulsive disorder, ${ }^{62,63}$ and deficient value-guided response selection in substance addiction have been reported. ${ }^{64}$ These regional alterations may reflect network-level dysfunctions in striatofrontal circuits, specifically the lateral OFC-caudate pathway engaged in attentional shifting and cognitive flexibility. ${ }^{15}$ The fNIRS-assisted regulatory control over the 1OFC may promote normalization of aberrant neural activation and promote functional recovery in psychiatric populations.

Examining the training effects on behavioral domains associated with the $1 \mathrm{OFC}$ revealed some evidences for the functional relevance of the training. Although no effects on accuracy in the set-shifting task were observed, the real-time OFC NF group 
demonstrated slightly faster reaction times for correct responses as compared to the sham NF group. The OFC critically contributes to flexible behavioral adaptations, with the lateral region supporting adaptation to changing reward contingencies during reversal learning. ${ }^{16-20,65}$ However, in the present study, regionalspecific modulation of the $1 \mathrm{OFC}$ did not affect the acquisition of the dimensional shifts per se. Despite several human imaging studies suggesting a role of the OFC in cognitive flexibility, ${ }^{23,66}$ previous animal studies demonstrated that regional-specific lesions of the lateral OFC did not critically disrupt set-shifting performance, which may explain the lack of strong performance effects on the set-shifting paradigm in the present study. ${ }^{20,67}$ Despite a lack of between-group differences in the subjective liking of the task, an exploratory correlational analysis revealed that stronger training-induced $1 \mathrm{OFC}$ increases in the real-time OFC NF group associated with higher post-training liking ratings, whereas no such association was observed in the sham NF group. These findings are in line with a previous report on a positive association between IOFC activation and subjective liking during a social reward paradigm ${ }^{36}$ and the important role of the $1 \mathrm{OFC}$ in processing reward-related outcome expectancies. ${ }^{68}$

The present findings need to be interpreted in the context of limitations. First, to reduce variance related to sex differences or effects of menstrual cycle on OFC-related functions and neural activity, the present proof-of-concept study focused on male participants (for a similar approach, see Zhao et al. ${ }^{6}$ ). The generalizability of the present results to female participants and potential sex differences thus needs to be examined in future studies. Second, despite some evidence for the effects of lOFC NF training on the behavioral level, the between-group comparisons of the primary behavioral outcomes failed to reach statistical significance. The lack of robust effects may be explained in terms of a low sensitivity of the set-shifting paradigm for activation changes in the lateral OFC. Moreover, the present study employed a single training session and more intense training schedules may be necessary to produce robust behavioral effects. Third, the present proof-of-concept study aimed at determining the feasibility of fNIRS-assisted training as a strategy to modulate the lOFC. In the context of the present study, the decision to focus on the right hemisphere was based on a previous human lesion study, suggesting a critical role of the right OFC in cognitive flexibility. ${ }^{69}$ However, the right $1 \mathrm{OFC}$ region in the present study included a total of four channels and future studies may consider initially employing a pretraining functional localization paradigm that allows to determine domain-specific and individualized training channels, which may further improve the behavioral effects of the training. Fourth, we did not observe evidence for differences in cognitive effort or stress between the training groups; however, future studies should consider to simultaneously acquire physiological measures such as heart rate or skin conductance responses to account for unspecific effects of training-associated stress or mental effort on the fNIRS signal. Fifth, no changes in the deoxy-Hb signal were observed over the course of the training runs. Previous studies observed a negative association between the oxy-Hb and the deoxy-Hb signals, particularly during motor processing. ${ }^{70}$ However, depending on the tasks and cortical regions assessed, the negative association has not been consistently observed, such that some studies reported small or no changes in the deoxy-Hb signal even when the oxy- $\mathrm{Hb}$ signal increased, including prefrontal regions during attentiondemanding and cognitive control tasks. ${ }^{71,72}$ In comparison, increases in the oxy-Hb signal have been more consistently observed during active task engagement and changes may be larger than those in the deoxy-Hb signal. ${ }^{73}$ On the other hand, the negative association between the deoxy-Hb and the oxy-Hb signals is influenced by noise in the data, with increasing noise leading to a lack of the (expected) negative association between the signals. ${ }^{74}$ The lack of a negative association in the present study strongly suggests that future NF studies should consider employing better control of potential noise sources in the data, ${ }^{75}$ such as simultaneous acquisition of head motion indices as well as additional monitoring of physiological signals such as cardiovascular indices. Finally, previous studies using fMRI-informed NF recently demonstrated that participants can maintain regulatory control for several days and in the absence of feedback. ${ }^{4,6}$ The present proof-of-concept study did not include a follow up or maintenance session and thus the maintenance of fNIRSassisted 1OFC regulatory control and its independence from online feedback remain to be determined in future studies.

In summary, the present findings demonstrate that real-time fNIRS-informed NF training of the OFC is feasible and that this approach allows participants to volitionally increase activation in this region. Given the high clinical relevance of altered OFC activity in psychiatric disorders, fNIRS-informed training of this region may represent a promising strategy to normalize OFC function in these disorders.

\section{Disclosures}

The authors declare no competing interests.

\section{Acknowledgments}

This work was supported by grants from the National Natural Science Foundation of China (NSFC) (Grant Nos. 91632117, 31530032, and 61431002); Fundamental Research Funds for the Central Universities (ZYGX2015Z002); Science, Innovation and Technology Department of the Sichuan Province (2018JY0001).

\section{References}

1. N. Birbaumer, S. Ruiz, and R. Sitaram, "Learned regulation of brain metabolism," Trends Cognit. Sci. 17(6), 295-302 (2013).

2. R. T. Thibault et al., "Neurofeedback, self-regulation, and brain imaging: clinical science and fad in the service of mental disorders," Psychother. Psychosom. 84(4), 193-207 (2015).

3. T. Watanabe et al., "Advances in fMRI real-time neurofeedback," Trends Cognit. Sci. 21(12), 997-1010 (2017).

4. S. Yao et al., "Voluntary control of anterior insula and its functional connections is feedback-independent and increases pain empathy," Neuroimage 130, 230-240 (2016).

5. K. D. Young et al., "Real-time functional magnetic resonance imaging amygdala neurofeedback changes positive information processing in major depressive disorder," Biol. Psychiatry 82(8), 578-586 (2017).

6. Z. Zhao et al., "Real-time functional connectivity-informed neurofeedback of amygdala-frontal pathways reduces anxiety," Psychother. Psychosom. 88(1), 5-15 (2019).

7. K. D. Young et al., "Randomized clinical trial of real-time fMRI amygdala neurofeedback for major depressive disorder: effects on symptoms and autobiographical memory recall," Am. J. Psychiatry 174(8), 748-755 (2017).

8. X. Zhang et al., "Single subject task-related BOLD signal artifact in a real-time fMRI feedback paradigm," Hum. Brain Mapping 32(4), 592-600 (2011).

9. J. G. Ojemann et al., "Anatomic localization and quantitative analysis of gradient refocused echo-planar fMRI susceptibility artifacts," Neuroimage 6(3), 156-167 (1997). 
10. D. Scheinost et al., "Orbitofrontal cortex neurofeedback produces lasting changes in contamination anxiety and resting-state connectivity," Transl. Psychiatry 3(4), e250 (2013).

11. N. K. Logothetis et al., "Neurophysiological investigation of the basis of the fMRI signal," Nature 412(6843), 150-157 (2001).

12. A. C. Ehlis et al., "Near-infrared spectroscopy as a new tool for neurofeedback training: applications in psychiatry and methodological considerations," Jpn. Psychol. Res. 60(4), 225-241 (2018).

13. L. R. Trambaiolli et al., "Functional near-infrared spectroscopybased affective neurofeedback: feedback effect, illiteracy phenomena, and whole-connectivity profiles," Neurophotonics 5(3), 035009 (2018).

14. M. L. Kringelbach and E. T. Rolls, "The functional neuroanatomy of the human orbitofrontal cortex: evidence from neuroimaging and neuropsychology," Prog. Neurobiol. 72(5), 341-372 (2004).

15. T. W. Robbins et al., "Neurocognitive endophenotypes of impulsivity and compulsivity: towards dimensional psychiatry," Trends Cognit. Sci. 16(1), 81-91 (2012).

16. G. Schoenbaum et al., "Does the orbitofrontal cortex signal value?" Ann. N.Y. Acad. Sci. 1239(1), 87-99 (2011).

17. I. Bohn, C. Giertler, and W. Hauber, "Orbital prefrontal cortex and guidance of instrumental behaviour in rats under reversal conditions," Behav. Brain Res. 143(1), 49-56 (2003).

18. Y. Chudasama and T. W. Robbins, "Dissociable contributions of the orbitofrontal and infralimbic cortex to pavlovian autoshaping and discrimination reversal learning: further evidence for the functional heterogeneity of the rodent frontal cortex," J. Neurosci. 23(25), 8771-8780 (2003).

19. J. Kim and M. E. Ragozzino, "The involvement of the orbitofrontal cortex in learning under changing task contingencies," Neurobiol. Learn. Memory 83(2), 125-133 (2005).

20. K. Mcalonan and V. J. Brown, "Orbital prefrontal cortex mediates reversal learning and not attentional set shifting in the rat," Behav. Brain Res. 146(1-2), 97-103 (2003).

21. G. Schoenbaum, "Lesions of orbitofrontal cortex and basolateral amygdala complex disrupt acquisition of odor-guided discriminations and reversals," Learn. Memory 10(2), 129-140 (2003).

22. G. Schoenbaum et al., "Orbitofrontal lesions in rats impair reversal but not acquisition of go, no-go odor discriminations," Neuroreport 13(6), 885-890 (2002)

23. A. Hampshire and A. M. Owen, "Fractionating attentional control using event-related fMRI," Cereb. Cortex 16(12), 1679-1689 (2006).

24. J. P. O'Doherty et al., "Neural responses during anticipation of a primary taste reward," Neuron 33(5), 815-826 (2002).

25. F. S. Arana et al., "Dissociable contributions of the human amygdala and orbitofrontal cortex to incentive motivation and goal selection," J. Neurosci. 23(29), 9632-9638 (2003).

26. L. J. Norman et al., "Structural and functional brain abnormalities in attention-deficit/hyperactivity disorder and obsessive-compulsive disorder," JAMA Psychiatry 73(8), 815-825 (2016).

27. L. Schmaal et al., "Cortical abnormalities in adults and adolescents with major depression based on brain scans from 20 cohorts worldwide in the ENIGMA major depressive disorder working group," Mol. Psychiatry 22(6), 900-909 (2017).

28. B. Becker and C. Montag, "Opinion: real-time fMRI neurofeedback and the application of the neuropeptide oxytocin as promising new treatment approaches in internet addiction?" in Internet Addiction, Studies in Neuroscience, Psychology and Behavioral Economics, C. Montag and M. Reuter, Eds., Springer, Cham, pp. 311-321 (2017).

29. A. C. Murphy and D. S. Bassett, "A network neuroscience of neurofeedback for clinical translation," Curr. Opin. Biomed. Eng. 1, 63-70 (2017).

30. M. L. Whittal, D. S. Thordarson, and P. D. Mclean, "Treatment of obsessive-compulsive disorder: cognitive behavior therapy vs. exposure and response prevention," Behav. Res. Ther. 43(12), 1559-1576 (2005).

31. A. A. Alegria et al., "Real-time fMRI neurofeedback in adolescents with attention deficit hyperactivity disorder," Hum. Brain Mapping 38(6), 3190-3209 (2017).

32. S. E. Kober et al., "Near-infrared spectroscopy based neurofeedback training increases specific motor imagery related cortical activation compared to sham feedback," Biol. Psychol. 95(1), 21-30 (2014).
33. M. Mihara et al., "Neurofeedback using real-time near-infrared spectroscopy enhances motor imagery related cortical activation," PLoS One 7(3), e32234 (2012).

34. S. M. H. Hosseini et al., "Task-based neurofeedback training: a novel approach toward training executive functions," Neuroimage 134, 153-159 (2016).

35. M. J. Hofmann et al., "Occipital and orbitofrontal hemodynamics during naturally paced reading: an fNIRS study," Neuroimage 94(7), 193-202 (2014).

36. Q. Li et al., "Foot massage evokes oxytocin release and activation of orbitofrontal cortex and superior temporal sulcus," Psychoneuroendocrinology 101, 193-203 (2019).

37. M. L. Schroeter et al., "Prefrontal activation due to Stroop interference increases during development—an event-related fNIRS study," Neuroimage 23(4), 1317-1325 (2004).

38. L. H. Ernst et al., "Prefrontal correlates of approach preferences for alcohol stimuli in alcohol dependence," Addict. Biol. 19(3), 497-508 (2014).

39. J. C. Dreher et al., "Menstrual cycle phase modulates reward-related neural function in women," Proc. Natl. Acad. Sci. U.S.A. 104(7), 2465-2470 (2007).

40. R. T. Thibault et al., "Neurofeedback with fMRI: a critical systematic review," Neuroimage 172, 786-807 (2018).

41. P. Sepulveda et al., "How feedback, motor imagery, and reward influence brain self-regulation using real-time fMRI," Hum. Brain Mapping 37(9), 3153-3171 (2016).

42. M. J. Van der Molen et al., "Attentional set-shifting in fragile $\mathrm{X}$ syndrome," Brain Cognit. 78(3), 206-217 (2012).

43. H. F. Ieong and Z. Yuan, "Abnormal resting-state functional connectivity in the orbitofrontal cortex of heroin users and its relationship with anxiety: a pilot fNIRS study," Sci. Rep. 7, 46522 (2017).

44. H. Jasper, "Report of the committee on methods of clinical examination in electroencephalography," Electroencephalogr. Clin. Neurophysiol. 10, 370-375 (1958).

45. N. Liu et al., "Optical-imaging-based neurofeedback to enhance therapeutic intervention in adolescents with autism: methodology and initial data," Neurophotonics 4(1), 011003 (2016).

46. P. Lapborisuth et al., "Neurofeedback-based functional near-infrared spectroscopy upregulates motor cortex activity in imagined motor tasks," Neurophotonics 4(2), 021107 (2017).

47. A. M. Marx et al., "Near-infrared spectroscopy (NIRS) neurofeedback as a treatment for children with attention deficit hyperactivity disorder (ADHD)—a pilot study," Front. Hum. Neurosci. 8, 1038 (2015).

48. L. Duan et al., "Cross-brain neurofeedback: scientific concept and experimental platform," PLoS One 8(5), e64590 (2013).

49. Statistical Parametric Mapping, SPM, https://www.fil.ion.ucl.ac.uk/ $\mathrm{spm} /(25$ May 2019).

50. J. C. Ye et al., "NIRS-SPM: statistical parametric mapping for nearinfrared spectroscopy," Neuroimage 44(2), 428-447 (2009).

51. T. Fekete et al., "The NIRS analysis package: noise reduction and statistical inference," PLoS One 6(9), e24322 (2011).

52. X. Cheng, X. Li, and Y. Hu, "Synchronous brain activity during cooperative exchange depends on gender of partner: a fNIRS-based hyperscanning study," Hum. Brain Mapping 36(6), 2039-2048 (2015).

53. S. M. Coyle, T. E. Ward, and C. M. Markham, "Brain-computer interface using a simplified functional near-infrared spectroscopy system," J. Neural Eng. 4(3), 219-226 (2007).

54. H. Yanagisawa et al., "Acute moderate exercise elicits increased dorsolateral prefrontal activation and improves cognitive performance with Stroop test," Neuroimage 50(4), 1702-1710 (2010).

55. T. J. Huppert, "Commentary on the statistical properties of noise and its implication on general linear models in functional near-infrared spectroscopy," Neurophotonics 3(1), 010401 (2016).

56. M. Kirsch et al., "Real-time functional magnetic resonance imaging neurofeedback can reduce striatal cue-reactivity to alcohol stimuli," Addict. Biol. 21(4), 982-992 (2016).

57. D. Watson, L. A. Clark, and A. Tellegen, "Development and validation of brief measures of positive and negative affect: the PANAS scales," J. Pers. Soc. Psychol. 54(6), 1063-1070 (1988).

58. C. D. Spielberger et al., Manual for the State-Trait Anxiety Inventory, Consulting Psychologists Press, Palo Alto, California (1983). 
59. A. T. Beck, R. A. Steer, and G. K. Brown, Manual for the Beck Depression Inventory-II, Psychological Corporation, San Antonio, Texas (1996).

60. J. H. Patton, M. S. Stanford, and E. S. Barratt, "Factor structure of the Barratt impulsiveness scale," J. Clin. Psychol. 51(6), 768-774 (1995).

61. G. A. Rousselet and R. R. Wilcox, "Reaction times and other skewed distributions: problems with the mean and the median," BioRxiv 383935 (2019).

62. J. C. Britton et al., "Cognitive inflexibility and frontal-cortical activation in pediatric obsessive-compulsive disorder," J. Am. Acad. Child Adolesc. Psychiatry 49(9), 944-953 (2010).

63. S. R. Chamberlain et al., "Orbitofrontal dysfunction in patients with obsessive-compulsive disorder and their unaffected relatives," Science 321(5887), 421-422 (2008).

64. R. C. Decharms et al., "Learned regulation of spatially localized brain activation using real-time fMRI," Neuroimage 21(1), 436-443 (2004).

65. R. Rygula et al., "Differential contributions of the primate ventrolateral prefrontal and orbitofrontal cortex to serial reversal learning," J. Neurosci. 30(43), 14552-14559 (2010).

66. R. Elliott, R. J. Dolan, and C. D. Frith, "Dissociable functions in the medial and lateral orbitofrontal cortex: evidence from human neuroimaging studies," Cereb. Cortex 10(3), 308-317 (2000).

67. E. A. Chase, D. S. Tait, and V. J. Brown, "Lesions of the orbital prefrontal cortex impair the formation of attentional set in rats," Eur. J. Neurosci. 36(3), 2368-2375 (2012).

68. G. Schoenbaum, M. R. Roesch, and T. A. Stalnaker, "Orbitofrontal cortex, decision-making and drug addiction," Trends Neurosci. 29(2), 116-124 (2006).

69. S. M. Levens et al., "Role of the left amygdala and right orbitofrontal cortex in emotional interference resolution facilitation in working memory," Neuropsychologia 49, 3201-3212 (2011).
70. L. Holper and M. Wolf, "Single-trial classification of motor imagery differing in task complexity: a functional near-infrared spectroscopy study," J. NeuroEng. Rehabil. 8(1), 34 (2011).

71. A. C. Ehlis et al., "Reduced lateral prefrontal activation in adult patients with attention-deficit/hyperactivity disorder (ADHD) during a working memory task: a functional near-infrared spectroscopy (fNIRS) study," J. Psychiatr. Res. 42(13), 1060-1067 (2008).

72. Y. Monden et al., "Right prefrontal activation as a neuro-functional biomarker for monitoring acute effects of methylphenidate in ADHD children: an fNIRS study," NeuroImage 1(1), 131-140 (2012).

73. T. Yamamoto and T. Kato, "Paradoxical correlation between signal in functional magnetic resonance imaging and deoxygenated haemoglobin content in capillaries: a new theoretical explanation," Phys. Med. Biol. 47(7), 1121-1141 (2002).

74. X. Cui, S. Bray, and A. L. Reiss, "Functional near infrared spectroscopy (NIRS) signal improvement based on negative correlation between oxygenated and deoxygenated hemoglobin dynamics," Neuroimage 49(4), 3039-3046 (2010).

75. I. Tachtsidis and F. Scholkmann, "False positives and false negatives in functional near-infrared spectroscopy: issues, challenges, and the way forward," Neurophotonics 3(3), 031405 (2016).

Benjamin Becker received his $\mathrm{PhD}$ in psychology from the Heinrich Heine University Duesseldorf (Germany) and currently, he is a full professor at the University of Electronic Science and Technology in Chengdu (China) and head of the neuSCAN Laboratory. His research combines neuroimaging with pharmacological and closedloop neuromodulation approaches to develop novel treatment for psychiatric disorders.

Biographies of the other authors are not available. 